\title{
Adaptive Ultrasound Doppler Velocimeter for the investigation of turbulent flows in liquid metals
}

\author{
Kevin Mäder, Richard Nauber, Hannes Beyer, Arne Klaß, Christian Kupsch, Norman Thieme, \\ Lars Büttner, Jürgen Czarske \\ Laboratory of Measurement and Sensor System Techniques, Faculty of Electrical and Computer \\ Engineering, School of Engineering, Technische Universität Dresden, 01062 Dresden, Germany \\ e-mail: kevin.maeder@tu-dresden.de
}

\begin{abstract}
In various industrial processes involving liquid metals time-dependent magnetic fields are used for flow control, e. g. during the solidification process of silicon to improve the quality and efficiency of wafers. In order to investigate the interactions between conductive fluids and spatiotemporally varying magnetic fields numerical simulations are performed. The numerical models are verified at model experiments with low-melting alloys. A suitable measurement technique for flow mapping in such model experiments is ultrasound Doppler velocimetry (UDV). We present the Phased Array Ultrasound Doppler Velocimeter (PAUDV), which extends the conventional UDV with the ability to dynamically steer and focus the acoustic field. The custom host software for control and signal processing allows to conduct wide range of different experiments, e.g. two-point correlation in turbulent flows or the measurement of two velocity components with a single acoustic access. Experimental results from $2 \mathrm{~d}-2 \mathrm{c}$ measurements and acquired two-point correlations are shown.
\end{abstract}

Keywords: Ultrasound Doppler Velocimetry, Phased Array, Flow Mapping, Liquid Metals

\section{Introduction}

In industrial processes involving molten metals quality, yield and energy efficiency can be improved by flow control. A promising approach is to affect the flow of electrically conductive fluids by using spatiotemporally varying magnetic fields. In the research field of magnetohydrodynamics (MHD) a better understanding of the relations between magnetic fields, induced Lorentz forces and their effect on conductive fluids is a main goal. Numerical models are used to investigate that complex interaction. For validation and refinement, the numerical simulations have to be accompanied by model experiments which are typically conducted using metal alloys with a low melting point. As an appropriate instrumentation system for multi plane, multi component flow mapping of such opaque liquids, systems like the Ultrasound Array Doppler Velocimeter (UADV) are available $[1,2]$. While this system offers a spatial resolution of up to $3 \mathrm{~mm}$ [3], for some experiments a higher resolution is desired in order to be able to resolve smaller flow structures. To address these experiments, ultrasound phased array beamforming [4] is a promising approach. It offers the possibility to dynamically focus and steer the sound field, therefore a higher spatial resolution can be achieved. This technique is widely used in multiple areas such as radar [5], ultrasound testing [6] or medicine [7]. It employs ultrasound transducer arrays with multiple elements arranged in a line or matrix. The transducer elements have sub-wavelength dimension in the dimensions where beamforming operation is applicable. This is required to make sure that every element transmits a spherical or cylindrical wave respectively. The sending pattern of each transducer element can be configured individually including a time delay. As the sound field of the whole array constitutes a superposition of all element emissions it can be shaped dynamically by specific time delay settings (see figure 1).

With the Phased Ultrasound Array Doppler Velocimeter we present a measurement system which combines ultrasound Doppler velocimetry with the Phased array technique to allow for high precision measurements in opaque fluids such as liquid metals. Providing individual programmable delays for all channels, transmit side beamforming is applicable. 


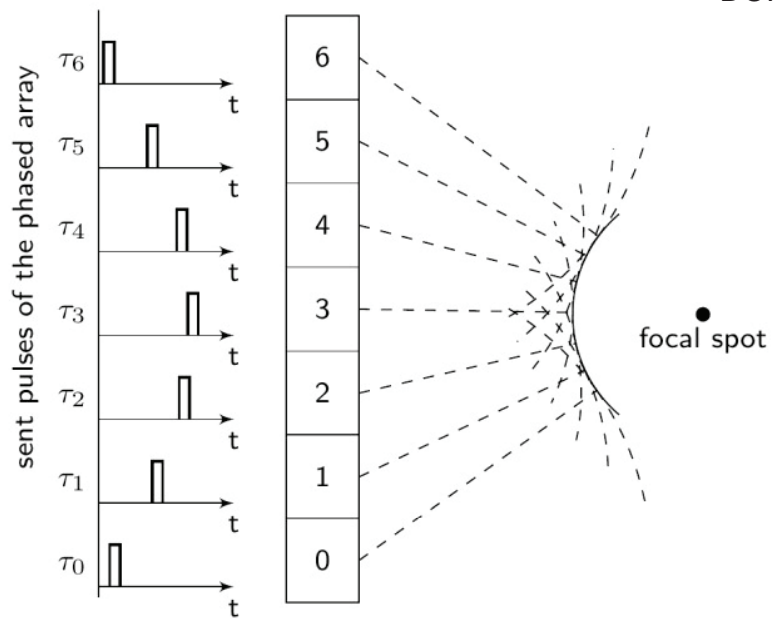

Figure 1: Principle of the phased array technique: The sound field of the array results from the superposition of signals of the single transducer elements. With individually delayed emission of the bursts the resulting sound field can be steered and focused dynamically.

Besides the hardware, a custom host software was developed. It offers the possibility for fine grained experiment definition, customized signal processing and beamforming on the receiving side.

\section{System Description}

The Phased Array Ultrasound Doppler Velocimeter (PAUDV) is designed as a measurement system for flow mapping using phased array ultrasound sensors with up to 256 channels in parallel. It uses the pulsedwave Doppler technique to measure the velocity of moving particles in a flow. Through the application of the phased array principle the sound field of the ultrasound arrays can be dynamically steered and focused (see figure 1). The system is able to transmit ultrasound bursts with a peak-to-peak amplitude of up to $200 \mathrm{~V}$, a frequency bandwidth of 1 to $10 \mathrm{MHz}$ and a pulse repetition frequency of up to $60 \mathrm{kHz}$. Delays for the pattern emission can specified with a resolution of $\Delta t=1.6 \mathrm{~ns}$. The modular design of the system allows for an easy adjustment of the hardware setup to the needs of the respective measurement task, e. g. usage of different or multiple ultrasound arrays.

Our custom host software includes functions covering the measurement process, from parameterizing functions for the setup to velocity estimators and result plotting. In addition, custom data structures are provided to represent the hardware setup and the experimental sequence. The latter describes the sequence of configurations and burst emission timings for the experiment. With the host software many different measurement modes are possible. This includes flow mapping with transmit or receive beamforming or simultaneous velocity measurements at multiple locations using time multiplexed focusing.

\section{Hardware architecture}

The PAUDV consists of a main controller module, up to eight 32-channel ultrasound transceiver modules, an analog signal backplane, a control backplane and an external acquisition unit. A block diagram and a picture of the system are shown in figure 2 . Each transceiver card contains an analog front end for receiving (RX) and a three voltage level driver for transmitting (TX) ultrasound signals. For transmission, delayed pulse patterns are generated by the TX beamformers for each channel individually. These burst signals are amplified by the high voltage pulse drivers and routed through the analog signal backplane to the respective transceiver elements of the ultrasound arrays. On the receiving side, signals are routed back through the corresponding $T / R$ switches to a preamplifier stage for low-noise signal conditioning. The analog signals are passed to the acquisition modules consisting of one FPGA (NI PXle 7965) and one A/D-converter module (NI 5752) each. In every module 32 channels are sampled simultaneously with a frequency of $32 \mathrm{MHz}$ and a quantization of 12 bit.

\section{Software architecture}

On the software side the PAUDV can be divided into the host software, the main controller firmware and a FPGA firmware (see figure 3). For control and signal processing the host software was implemented in the programming language Python. Through this software an experiment to conduct is described by a hardware setup model, an experimental sequence and a data processing procedure. For the hardware setup model, every module of the PAUDV system is represented by an object. Electrical interconnections between modules are mapped by connections between these objects thus reflecting signal paths. Using this structure every part of any signal 


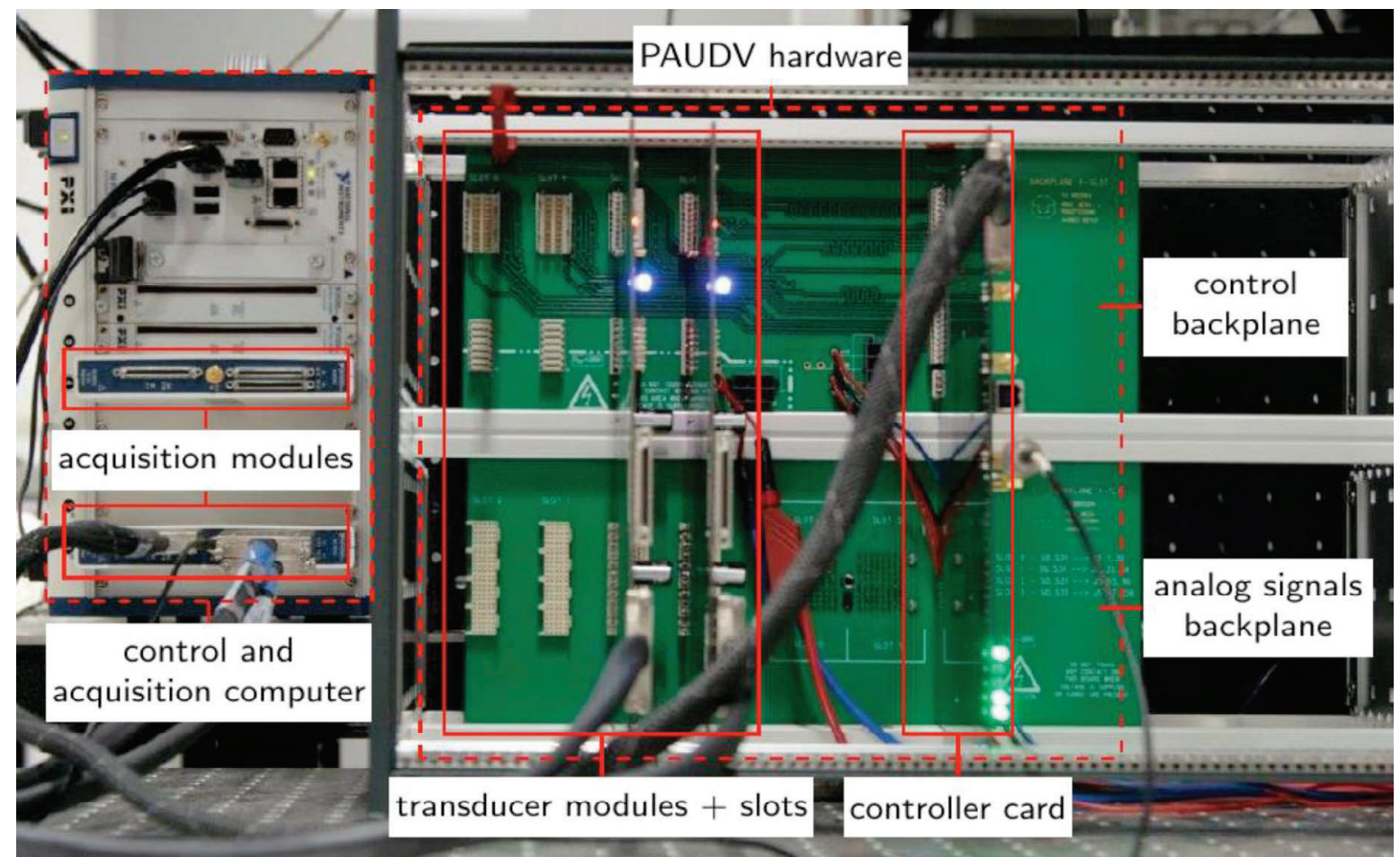

(a)

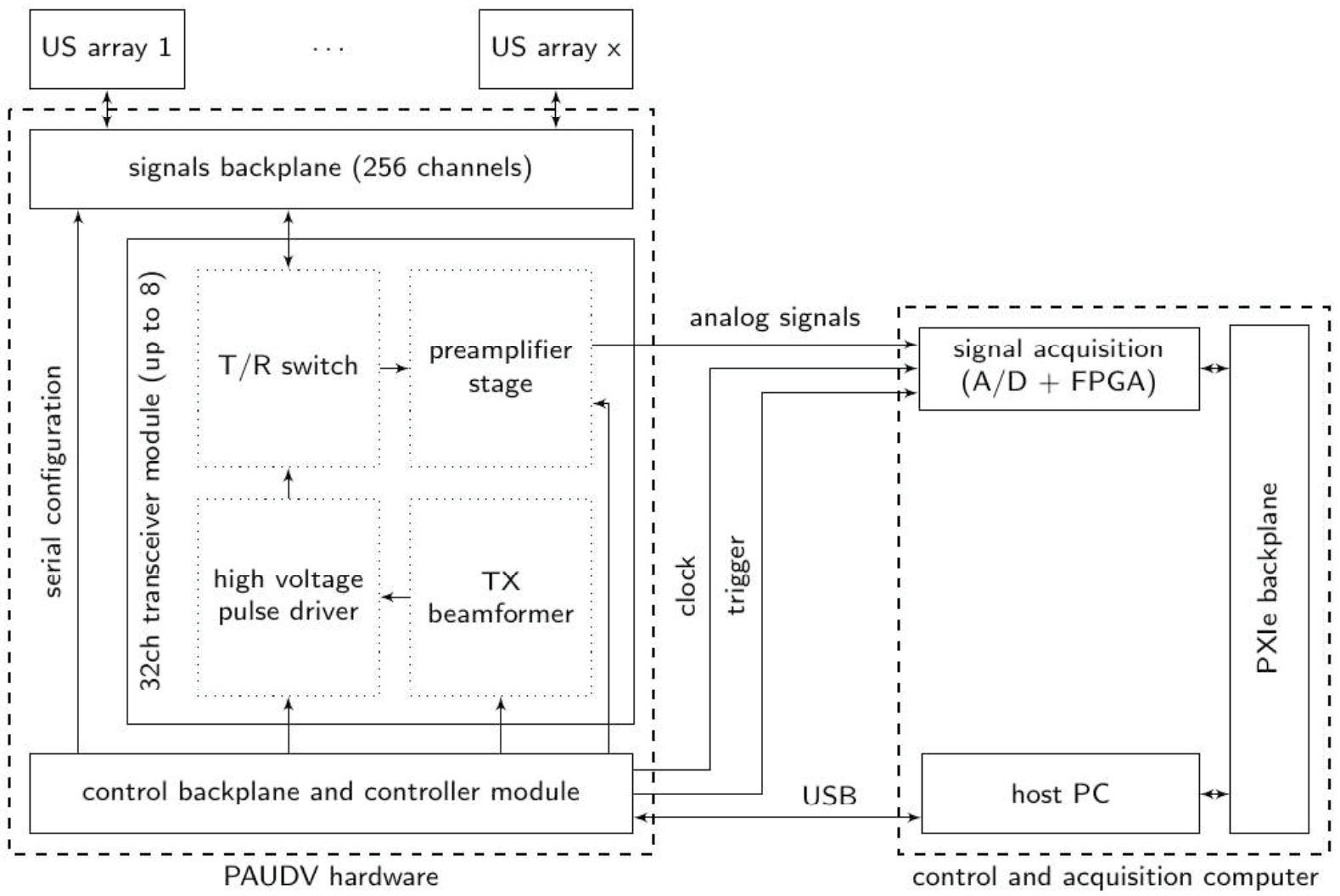

(b)

Figure 2: Picture (a) and block diagram (b) of the Phased Array Ultrasound Doppler Velocimeter (PAUDV): The PAUDV consists of a computer with acquisition modules and the custom PAUDV hardware. The latter is composed of the main controller module, transceiver modules, a control and an analog signals backplane. The current prototype can hold up to 4 transceiver cards and can be easily extended to use up to 8 cards. 

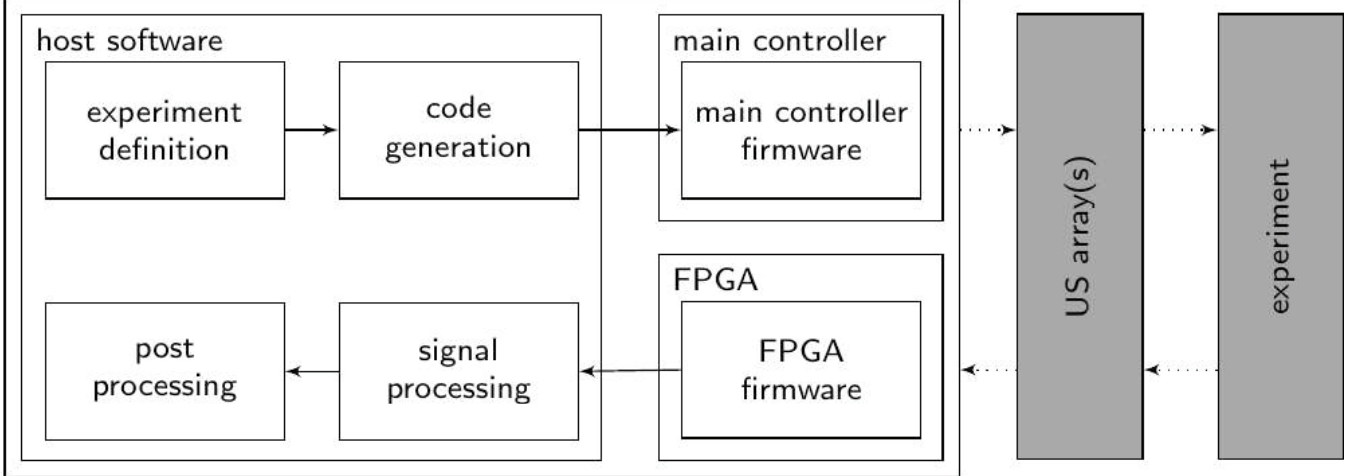

Figure 3: Software architecture of the PAUDV: Using the host software the experiment to conduct is described and a program for the main controller is generated. The generated program is run on the main controller of the PAUDV hardware. Captured signals are preprocessed by the FPGA. Signal and post processing of the data is done by the host software.

path can be referenced by their respective transceiver element object. Because the hardware setup is described separately from other experiment related information it enables the user to reuse described experiments with different hardware setups requiring only small changes.

The experimental sequence is created as a hierarchy of predefined segments. These predefined segments offered by the host software provide functionality for single velocity measurements as well as to group, interleave or loop over multiple segments. Each segment can be parameterized individually. This approach allows to specify a wide range of experiments in a structured manner. Conducting the experiment, the defined experimental sequence is compiled to an instruction list by the host software. These instructions are transferred to the main controller of the PAUDV hardware via an USB connection. This controller is a micro-controller based real-time system which executes the received programs to control all time-critical functionality of the PAUDV hardware. E.g. it sets the parameters on the transceiver cards such as time delays, pulse patterns and amplification gains according to the programmed sequence. On the acquisition side the digitized signals of the A/D-converter modules are preprocessed by the FPGA modules. With the current FPGA firmware time gain control can be applied. The resulting signal data is saved on the host PC. For signal and post processing of the acquired data the host software provides functions for filtering, receive beamforming, two-point correlation and velocity estimation. For the latter an algorithm based on an autocorrelation method [8] was

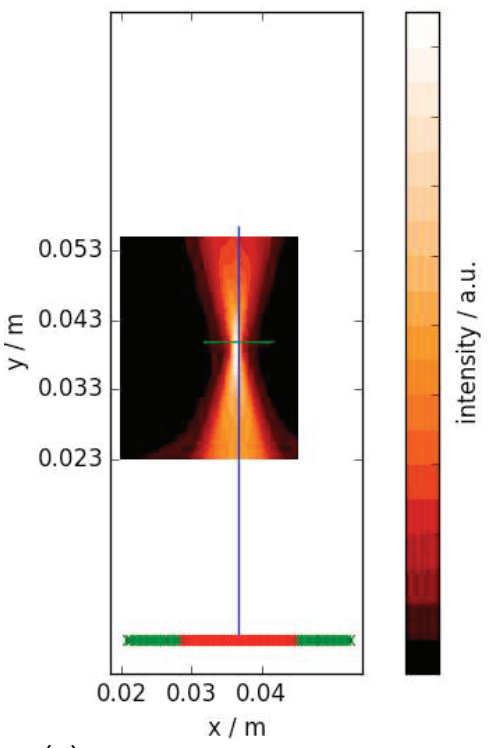

(a)

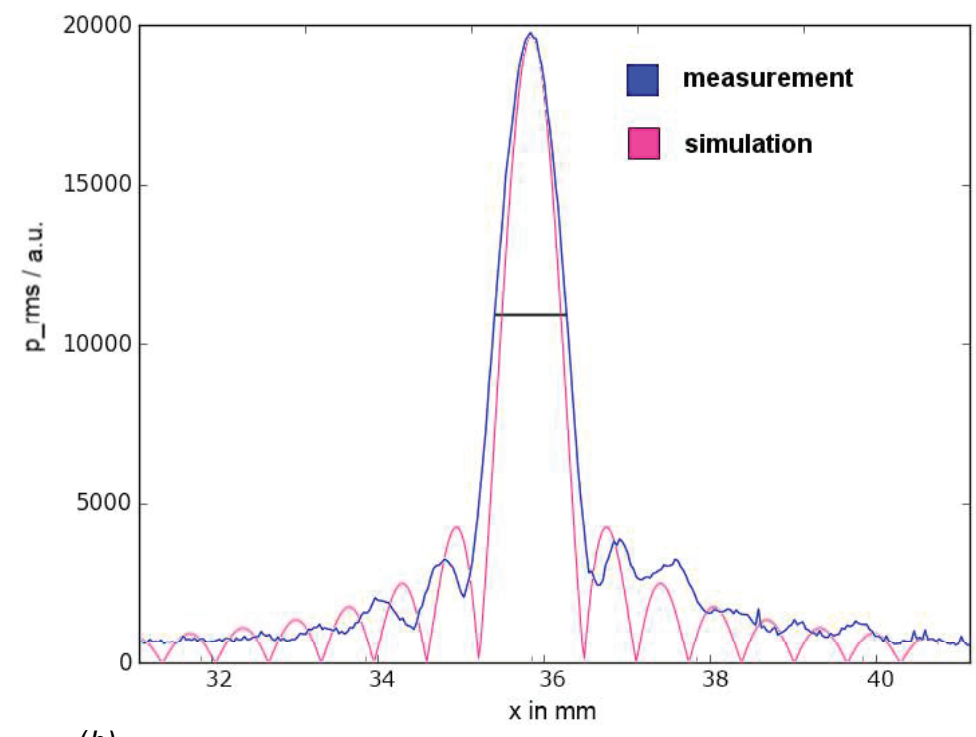

(b)

Figure 4: Measured sound field for a focus distance of $F=40 \mathrm{~mm}$ in front of the phased array sensor.

(a) plot of the measured sound field

(b) scan of the sound field through the focus point along the $x$-axis in comparison to the theoretical limit for the used transducer from a simulation. 


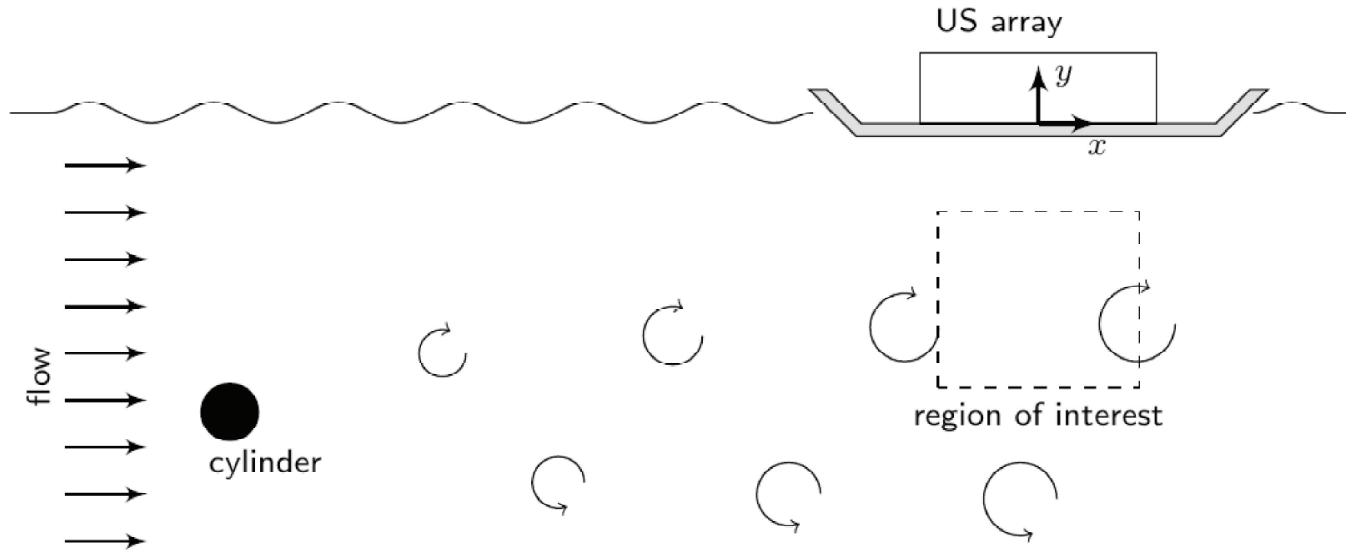

Figure 5: Setup of the conducted flow measurements: The laminar flow is disturbed by a cylinder. This causes a Kármán vortex street to develop. The phased array is positioned at the surface of the water.

implemented. Using the provided functions, the experimenter can define a data processing procedure which is run for every single velocity measurement of the experimental sequence. As these processing jobs are mostly independent of each other, parallel execution is supported to improve the runtime performance. Processing results are saved in a data container which provides an easy interface for further processing or result plotting.

\section{Measurement results}

To test the functionality of the system the resulting sound field of a focusing configuration with a distance of $y=F=40 \mathrm{~mm}$ between focus and the phased array sensor was characterized. This was done by scanning the region around the focus using a wire with a diameter of $d=120 \mu \mathrm{m}$ that was aligned along the z-axis. From the recorded sound field the spatial resolution in lateral direction was measured as the full width at half maximum of an intensity scan along the $x$-axis through the focal point. For the conducted experiment in water with an ultrasound frequency of $f=3 \mathrm{MHz}$ a spatial resolution of approx. $1 \mathrm{~mm}$ was determined (see figure 4).

To demonstrate the flow mapping capabilities, experiments in a rectangular water flow channel were conducted. There the Kármán vortex street in the wake flow of a cylinder was observed. The hardware setup for the conducted experiments is depicted in figure 5 . Two component flow mapping with one acoustical access was done using a cross beam method [9]. The phased array employed has 128 elements and a pitch of $0.5 \mathrm{~mm}$. It was positioned parallel to the flow direction.

Figure 6 shows an averaged two component flow mapping. In the figure the wake space which results from averaging over the counterrotating vortices behind the flow obstacle can

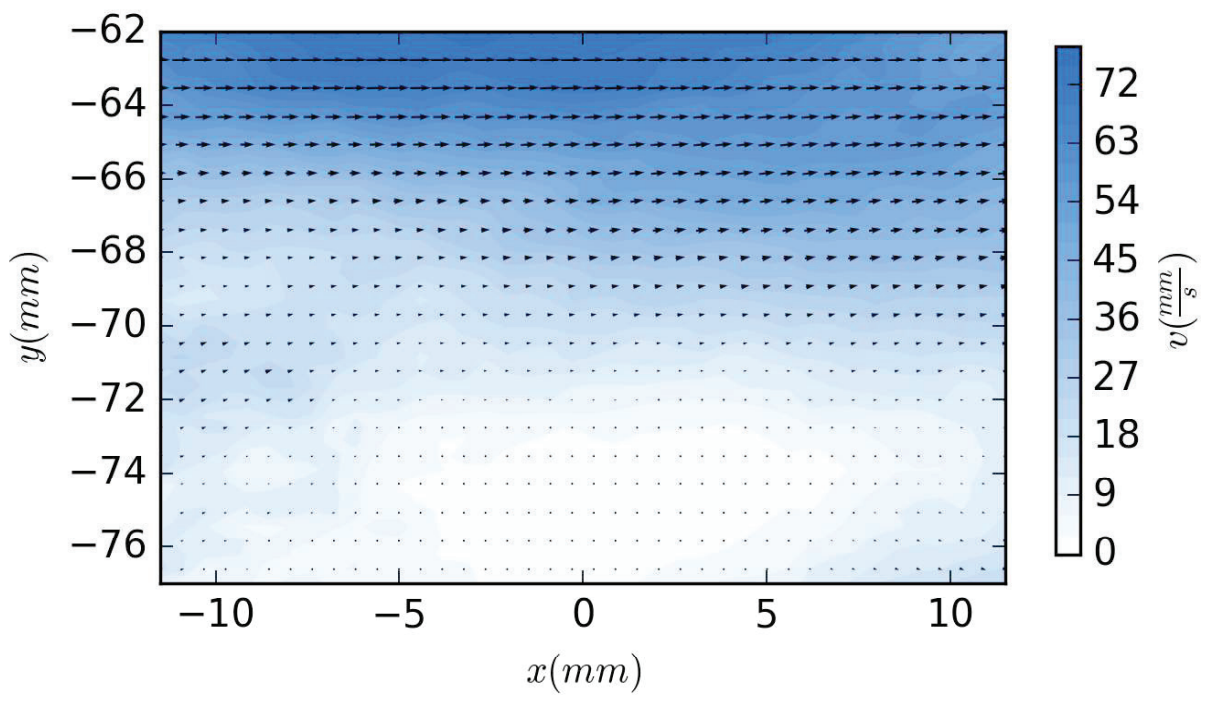

Figure 6: Averaged two component flow mapping of water behind a cylinder at $x=-43 \mathrm{~mm}, y=-72 \mathrm{~mm}$ : Absolute flow speed is indicated by the color while arrows point into direction of flow. The main flow is in positive $x$ direction. The ultrasound array is positioned with its center at $x=0, y=0$. The averaging time is 50 s. The plots depicts the upper bound of the wake space behind the cylinder. 

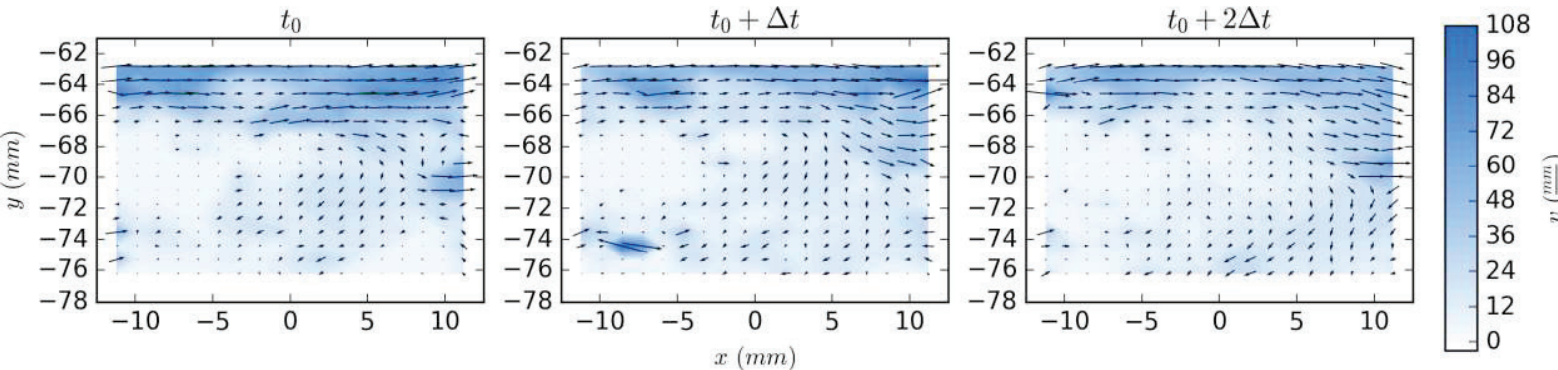

Figure 7: Velocity component in $y$-direction of the flow behind a cylinder $(x=-93 \mathrm{~mm}, y=-42 \mathrm{~mm}, \varnothing=10 \mathrm{~mm})$ at three consecutive times with $\Delta t=0.05 \mathrm{~s}$ : The ultrasound array is positioned with its center at $x=0, y=0$. The main flow is in positive $x$-direction. In the time series passing flow structures can be seen.

be identified. Results of a flow mapping experiment behind a cylinder for 3 consecutive times $(\Delta t=0.05 \mathrm{~s})$ are displayed in figure 7 . This time-resolved measurement shows the flow velocity behind a cylinder. In the figure the passing of a flow structure with a lower velocity is visible. In addition to flow mapping two-point correlations functions have been measured. Such measurements are done by quasisimultaneously measuring the velocity at two positions with a distance $\Delta x$ (see figure $8 a$ ) and correlating the two velocity series. Using these functions, length scales for the characterization of turbulent flows can be determined [10]. Figure $8 b$ depicts a result plot of a measurement with the PAUDV and an optical PIV system. The plot shows good agreement between the two measurements.

\section{Summary and Outlook}

Model experiment studies are necessary for research in MHD, as these are needed for a better understanding of the interaction between magnetic fields and conductive fluids. The modular PAUDV allows to instrument experiments in opaque liquids with a spatial resolution of under $1 \mathrm{~mm}$. In addition, high resolution two component scanning while needing only one acoustical access is possible.

With its 256 distinct channels transmit and receive beamforming is applicable for linear or two-dimensional arrays. A maximum pulse repetition frequency of $60 \mathrm{kHz}$ can be achieved which offers the possibility to adjust the time resolution as necessary.

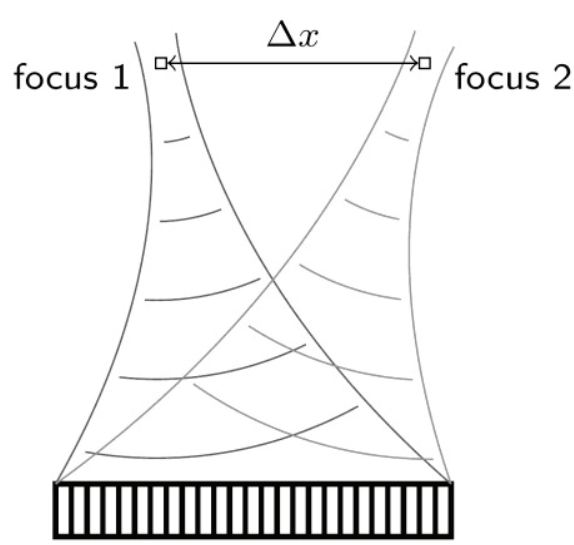

(a)

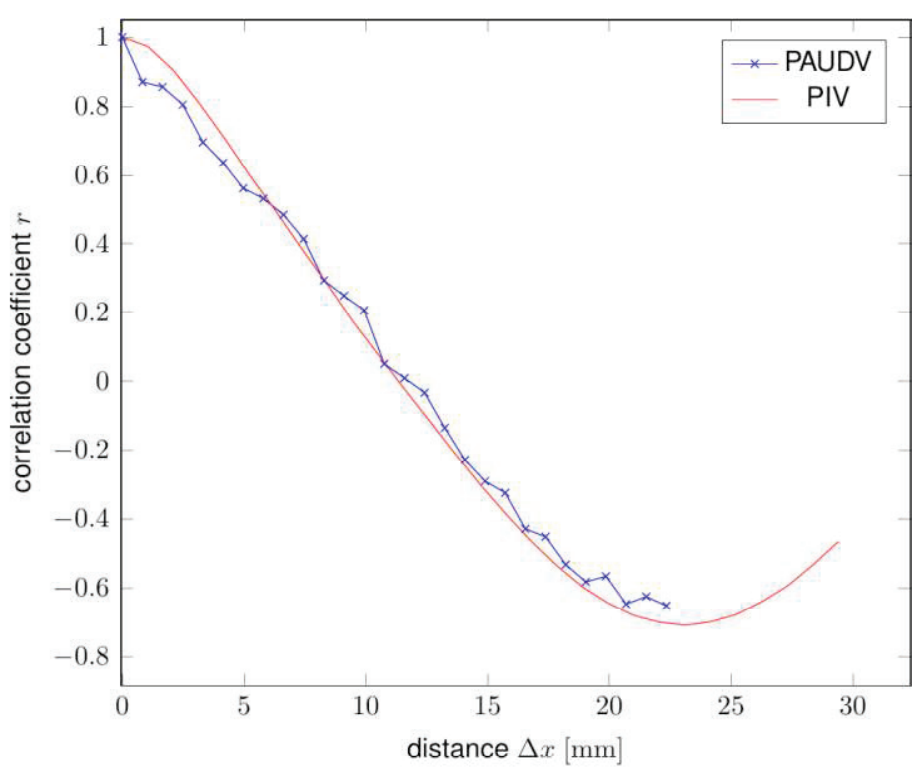

(b)

Figure 8: Principle (a) and result plot (b) of two-point correlation measurements: For two-point correlations velocity measurements are performed at two focal points with a distance of $\Delta x$ quasi-simultaneously. From these velocity series at two focus points a correlation coefficient is computed. Measurements with different distances $\Delta x$ yield velocity correlation functions. Plot (b) shows the results of a simultaneous measurement with the PAUDV and an optical PIV system. The measurement was conducted in the wake behind a cylinder (position: $x=-93 \mathrm{~mm}$, $y=-42 \mathrm{~mm} ; \varnothing=10 \mathrm{~mm})$ with focus 1 fixed at $(x=-11 \mathrm{~mm}, y=39 \mathrm{~mm})$ and focus 2 at $(x=-11 \mathrm{~mm}+\Delta x$, $y=39 \mathrm{~mm}$ ). 
A custom host software was implemented to simplify the control of the device and the respective data processing. Therefor the used hardware setup is modelled in software. In addition, the experimental sequence can be composed as a hierarchy of predefined segments allowing a variety of experiments.

In an experiment conducted in a water channel the capabilities of the system for $2 d-2 c$ measurements with one acoustical access or quasi-simultaneous measurements at multiple foci for two-point correlations were shown.

We plan to apply the system to room temperature models of the continuous steel casting process as well as semiconductor crystal growth experiments.

\section{Acknowledgements}

The research is supported by the Deutsche Forschungsgemeinschaft (DFG) within the grant DFG BU 2241/2-1. The financial support is gratefully acknowledged by the authors. Furthermore, we thank Sonaxis SA (Besançon, France) for the efforts manufacturing the custom ultrasound transducer.

\section{References}

[1] L. Büttner, R. Nauber, M. Burger, D. Räbiger, S. Franke, S. Eckert and J. Czarske, Measurement Science and Technology, 24(5):055302, 2013

[2] R. Nauber, M. Burger, M. Neumann, L. Büttner, K. Dadzis, K. Niemietz, O. Pätzold, and J. Czarske. Experiments in Fluids, 54(4), 2013

[3] M. Burger, R. Nauber, M. Neumann, L. Büttner, and J. Czarske, tm - Technisches Messen, 79(9):417-425, September 2012

[4] V. Schmitz, W. Müller, and G. Schäfer, Acoustical Imaging, 19: 545-551. Springer US, 1992

[5] P. Bradsell, IEE Tutorial Meeting on Phased Array Radar, London, 1989

[6] J. Gao, K. Wang and J. Sun, International Journal of Signal Processing, Image Processing and Pattern Recognition, 6(5), $71-78,2013$

[7] T. Szabo and P. Lewin, Journal of Ultrasound in Medicine, 32(4): 573-582, 2013

[8] T. Loupas, J.T. Powers, and R.W. Gill, Ultrasonics, Ferroelectrics, and Frequency Control, IEEE Transactions on, 42(4):672688, 1995

[9] B. Dunmire, K. W. Beach, K.-H. Labs, M. Plett, and D. E. Strandness Jr., Ultrasound in Medicine and Biology, 26(8):1213-1235, 2000

[10] H. Tennekes, J. Lumley, A first course in turbulence, MIT Press 1997 